\title{
Simulation design of a circular wave-guide phase equalizer
}

\author{
Yang Linchuan \\ Dept. Electromagnetic Wave and Antenna \\ Information Science and Technology Institute \\ Zhengzhou, China \\ qunshiyan1981@163.com
}

\author{
Zhang Dewei \\ Dept. Electromagnetic Wave and Antenna \\ Information Science and Technology Institute \\ Zhengzhou, China \\ zhdw3221@163.com
}

\author{
Zhou Dongfang \\ Dept. Electromagnetic Wave and Antenna \\ Information Science and Technology Institute \\ Zhengzhou, China \\ zhdfxd@yahoo.cn
}

\begin{abstract}
In this paper, theoretical analysis of phase equalization technology is carried on, and phase characteristics of the circular wave-guide loaded metal screws is studied. On this basis, a Ku-band circular wave-guide phase equalizer is proposed. Simulation analysis with the electromagnetic simulation software HFSS shows that the amount of the insertion phase shift can be effectively controlled by adjusting the parameters of the diameter and length of the circular wave-guide, the diameter, insertion depth, e number and spacing of the screws.
\end{abstract}

Keywords- Phase Equalizer; Circular Wave-guide; Metal Screws

\section{INTRODUCTION}

Phase distortion mainly comes from the high selective components, such as selective amplifiers, filters, etc. To solve the problem of phase fluctuation of these devices in working band, the feasible method is equilibrium technology used in the devices or outside the devices, thereby eliminating the phase distortion of the channel, this kind of method called phase equilibrium technology. In the link, the stronger amplitude selectivity of the device, the stronger the introduction of phase distortion, the greater the need to phase equalizer. For example, in the satellite communication system, the filters are often placed on the satellite, while the phase equalizers are in the satellite earth stations [1]. In addition to meet distortionless condition, it needs phase equalizer, in the broadband power synthesis technology [2], it also needs phase equilibrium technology. This is because that the phase consistency of the synthetic unit directly relates to the size of the synthetic power. Using phase equalizer to adjust the phase of each unit can reduce phase loss caused by the phase mismatch.

\section{THEORY OF THE PHASE EQUILIBRIUM TECHNOLOGY}

In the frequency domain, to make the signal without distortion transfer, the amplitude frequency characteristics of the network should be a constant unconnected with the frequency; Phase frequency characteristics should be a straight line over origin. Phase frequency characteristics of the channel are usually measured by group delay characteristics. When the signal without distortion transfer, the phase frequency characteristics of the channel are linear, that is a linear relationship between $\phi(\omega)$ and $\omega$. And the channel group delay is $\tau_{g}=-d \phi(\omega) / d \omega$, so the signal without distortion transfer, the group delay characteristics curve will be a level of straight line. The phase equalizer is based on the principle described above with a phase lag compensation techniques [3], when it is a small delay on the signal frequency, it should increase the delay. While the delay of the signal frequency is big, it should be appropriate to reduce the delay or no delay so that the group delay are basic consistent on each frequency point. The group delay characteristics of the channel at frequency domain are close to a horizontal straight line, as shown in Figure 1, thereby reducing the phase distortion of the channel, and improving the data transfer rate.

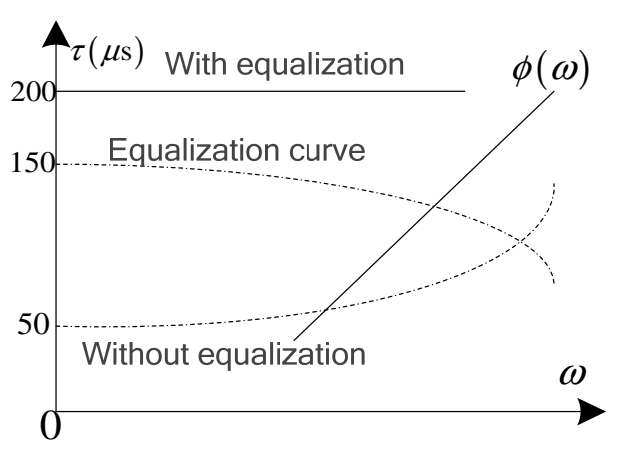

Fig. 1 Phase equilibrium principle diagram 


\section{CIRCULAR WAVEGUIDE LOADED METAL SCREWS PHASE CHARACTERISTIC ANALYSIS}

\section{A. Determination of the phase shift}

The circular waveguide loaded metal screws can achieve the phase shift on a particular frequency point, this structure can be used as a model of the phase equalizers. As shown in Figure 2, Metal screws through the circular waveguide wall symmetrically insert and arrange at equal intervals [4], each pair of screws can be used as a basic unit of the phase shift.

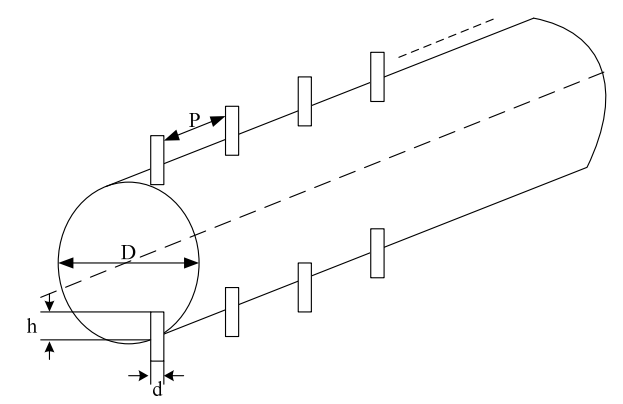

Fig. 2 Circular waveguide loaded multi screws diagram

As shown in Figure 3, for the structure of a pair of screws inserted into the circular waveguide, the arbitrary direction of the incident wave can be divided into two components $E_{V}$ and $E_{H}$ which are vertical with each other and have equal amplitude and phase. The equivalent circuit is shown in figure 4 (a), it can be further equivalent to a transmission line [5] as shown in figure 4 (b).

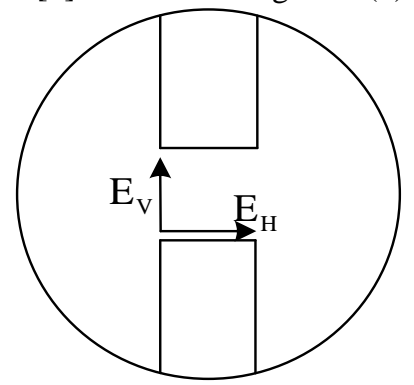

Fig. 3 Decomposition of the incident wave

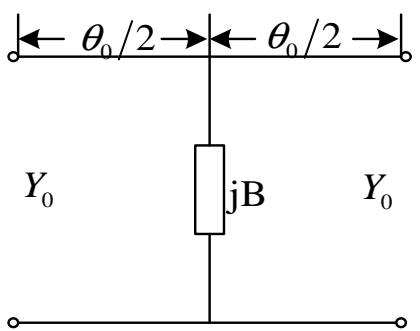

(a)

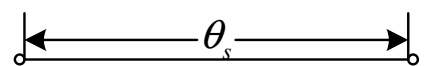

$Y_{s}$

(b)

Fig. 4 The equivalent circuit and the equivalent transmission line of the metal screws

It can be obtained by the microwave network theory

$$
\begin{aligned}
& {\left[\begin{array}{cc}
\cos \theta_{s} & j \frac{1}{Y_{s}} \sin \theta_{s} \\
j Y_{s} \sin \theta_{s} & \cos \theta_{s}
\end{array}\right]=\left[\begin{array}{cc}
\cos \frac{\theta_{0}}{2} & j \frac{1}{Y_{0}} \sin \frac{\theta_{0}}{2} \\
j Y_{0} \sin \frac{\theta_{0}}{2} & \cos \frac{\theta_{0}}{2}
\end{array}\right] \times} \\
& {\left[\begin{array}{cc}
1 & 0 \\
j B_{V, H} & 1
\end{array}\right] \times\left[\begin{array}{cc}
\cos \frac{\theta_{0}}{2} & j \frac{1}{Y_{0}} \sin \frac{\theta_{0}}{2} \\
j Y_{0} \sin \frac{\theta_{0}}{2} & \cos \frac{\theta_{0}}{2}
\end{array}\right]=} \\
& {\left[\begin{array}{cc}
\cos \theta_{0}-\frac{B_{V, H}}{2 Y_{0}} \sin \theta_{0} & j \frac{1}{Y_{0}}\left(\sin \theta_{0}-\frac{B_{V, H}}{Y_{0}} \sin ^{2} \frac{\theta_{0}}{2}\right) \\
j Y_{0}\left(\sin \theta_{0}+\frac{B_{V, H}}{Y_{0}} \cos ^{2} \frac{\theta_{0}}{2}\right) & \cos \theta_{0}-\frac{B_{V, H}}{2 Y_{0}} \sin \theta_{0}
\end{array}\right]}
\end{aligned}
$$

Wherein $\theta_{0}$ is the electrical length of a transmission line, $\theta_{s}$ is an equivalent electrical length of the equivalent transmission line, $B_{H}$ and $B_{V}$ are presented to be the susceptances of the wave in two directions respectively, $Y_{0}$ is the characteristics admittance of the waveguide. It can be obtained from the above equation

$$
\begin{aligned}
& \cos \theta_{s}=\cos \theta_{0}-\frac{B_{V, H}}{2 Y_{0}} \sin \theta_{0} \\
& \theta_{s}=\cos ^{-1}\left(\cos \theta_{0}-\frac{B_{V, H}}{2 Y_{0}} \sin \theta_{0}\right) \\
& \Delta \theta=\cos ^{-1}\left(\cos \theta_{0}-\frac{B_{H}}{2 Y_{0}} \sin \theta_{0}\right) \\
& -\cos ^{-1}\left(\cos \theta_{0}-\frac{B_{V}}{2 Y_{0}} \sin \theta_{0}\right)
\end{aligned}
$$

$\Delta \theta$ is the retardation which comes from $E_{V}$ and $E_{H}$ passing an circular wave-guide inserted a pair of screws, the phase difference after inserting $\mathrm{N}$ pairs of metal screws is

$$
\Delta \theta_{\text {总 }}=\sum_{\mathrm{i}=1}^{\mathrm{N}} \Delta \theta_{\mathrm{i}}
$$


Obviously, if the value of $\frac{B_{V}}{Y_{0}}$ and $\frac{B_{H}}{Y_{0}}$ are determined, it can be obtained a retardation. Adjusting the size of $\frac{B_{V}}{Y_{0}}$ and $\frac{B_{H}}{Y_{0}}$ can realize the phase shift regulation on various frequency points.

\section{B. Solving equivalent susceptance}

For the susceptance solution of a single metal screw, literature [6] gives a specific method. Firstly, 3(a) is regarded as a transmission line of an equivalent half wavelength, calculate the effect of changes of the transmission line accessing susceptance, and then compare with the effect of the tuning screws of the resonator, the susceptance of metal screws can be obtained by using the perturbation method, whereby

$$
\frac{B}{2 Y_{0}}=\frac{\pi}{2}\left(\frac{\lambda \mathrm{g}}{\lambda}\right)^{2} \mathrm{C} \int_{\Delta \mathrm{V}}\left[\frac{\varepsilon}{\mu}|\mathrm{E}|^{2}-|\mathrm{H}|^{2}\right] \mathrm{dV}
$$

$\mathrm{E}$ and $\mathrm{H}$ are the normalized field of the resonant cavity, $\int_{\mathrm{V}}|\mathrm{H}|^{2} \mathrm{dV}=1$; $\mathrm{c}$ is the field changes after inserted a screw, it is related to the size of the waveguide and frequency, and it is usually determined by experiment; $\Delta \mathrm{V}$ is the volume of the screw inserted into the waveguide.

$\mathrm{TE}_{111}$ mode is the lowest order mode of a cylindrical cavity, so this mode can be used to calculate a pair of metal screws' susceptance .

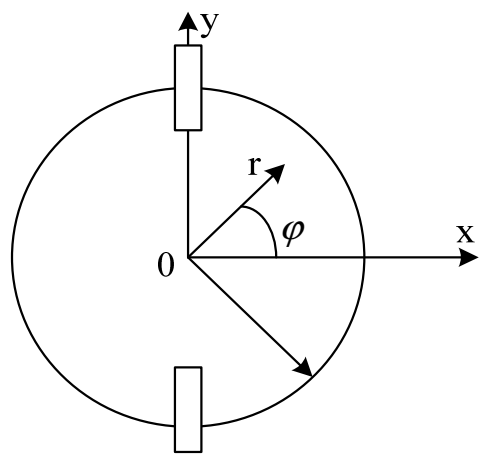

Fig. 5 Screw location diagram

Figure 5 shows the position of the screws, when the field are parallel to the screws, $\phi=\frac{\pi}{2}$, only component

$$
\mathrm{E}_{\mathrm{r}}=\mathrm{j} \frac{\omega \mu}{\mathrm{k}_{\mathrm{c}}} \frac{\mathrm{J}_{1}\left(\mathrm{k}_{\mathrm{c}} \mathrm{r}\right)}{\mathrm{k}_{\mathrm{r}}}
$$

Substitute (7) into (6), then get

$$
\frac{B_{V}}{Y_{0}}=\mathrm{K}_{1} \pi^{2} \frac{\lambda_{\mathrm{g}}}{\lambda^{2}} \frac{\mathrm{hr}_{0}^{2}}{\mathrm{a}^{2}}
$$

when the field are perpendicular to the screws, $\phi=0$, only component

$$
\mathrm{H}_{\mathrm{z}}=\mathrm{J}_{1}\left(\mathrm{k}_{\mathrm{c}} \mathrm{r}\right)
$$

Substitute (9) into (6), then get

$$
\frac{B_{\mathrm{H}}}{Y_{0}}=-\mathrm{K}_{2} \pi^{2} \frac{\lambda_{\mathrm{g}}}{\lambda_{\mathrm{c}}^{2}} \frac{\mathrm{hr}_{0}^{2}}{\mathrm{a}^{2}}
$$

Substitute (8) and (10) into (4), the phase difference $\Delta \theta$ can be obtained.

\section{DESIGN EXAMPLE}

According to the theory, a Ku-band (8GHz-8.5 GHz ) phase equalizer using a double row screws structure is designed. Take circular waveguide diameter $\mathrm{D}=22.4 \mathrm{~mm}$, metal screws diameter $\mathrm{d}=0.77 \mathrm{~mm}$, screw pitch $\mathrm{p}=5 \mathrm{~mm}$, screws insertion depth are shown in Table 1.

Table 1. Screw depth distribution

\begin{tabular}{|c|c|c|c|c|c|c|c|c|}
\hline $\mathrm{n}$ & 1 & 2 & 3 & 4 & 5 & 6 & 7 & 8 \\
\hline $\mathrm{H}(\mathrm{mm})$ & 2 & 1.5 & 1 & 0.5 & 0.5 & 1 & 1.5 & 2 \\
\hline
\end{tabular}

Using electromagnetic simulation software HFSS, the structure is shown in Figure (5). Simulation results show that this structure has good VSWR. Increasing screws pitch $\mathrm{P}$ can improve the matching, but the screw pitch $\mathrm{P}$ cannot be too long. When the penetration depth and the radius of the screws increase, the susceptance presents big, and the deeper the penetration depth, the narrower the band, the frequency response is not flat.

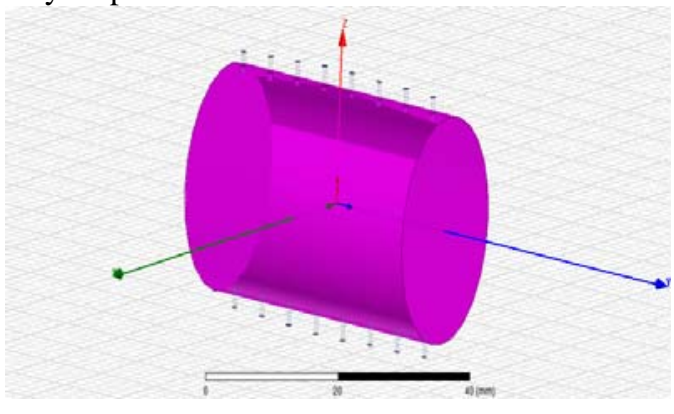

Fig. 6 HFSS simulation structure diagram

\section{CONCLUTIN}

In this paper, the phase equalizer is analyzed theoretically, and a kind of $\mathrm{Ku}$ band circular waveguide phase equalizer is designed. To design this kind of phase equalizer, the diameter $\mathrm{D}$, length of the circular wave-guide, the diameter $\mathrm{d}$, number, spacing and the insertion depth of the screws should be considered. The phase of corresponding frequency points can be adjusted by changing the parameter settings.

\section{REFERENCES}

[1] Chen Shuyuan, Yan Shaoshu. "Phase Shift Equalizer”. People's Posts and Telecommunications Press , 1984: 3-4.

[2] Xie Ning,Yang Jun,Sui YuXing. "Study of Pulse TWT Transmitters Power Combining Technology ". Journal of Modern Rader, 2007,29 (9): 87-88. 
[3] Chen Shuyuan, Yan Shaoshu. "Phase Shift Equalizer”. People’s Posts and Telecommunications Press , 1984: 159-161.

[4] Shi Yang,Shi ZhiDong,Li MingXiang,Xu Hao. "Design and Optimization of Waveguide Circular Polarizer Using $8 \mathrm{~mm}$ Adjustable Metal Screws". JOURNAL OF SHANGHAI UNIVERSITY(NATUAL SCIENCE), 2007, 13(2):134-136.

[5] Qiao Ben, Wu Tian. "Corrugated Gating Bandwidth Sharing Circular Polarizer ”. Journal of Microwave Research of Information Institute, 1975 .

[6] Sun HuiBao. "Analysis and Calculation of the Multi Screws Polarizer ”, Journal of Tracking Rader, 1978. 